\title{
Aven is dynamically regulated during Xenopus oocyte maturation and is required for oocyte survival
}

\author{
L O'Shea ${ }^{1}$, T Fair $^{2}$ and C Hensey ${ }^{*, 1}$
}

We have analyzed the expression and function of the cell death and cell cycle regulator Aven in Xenopus. Analysis of Xenopus Aven expression in oocytes and embryos revealed a band close to the predicted molecular weight of the protein $(36 \mathrm{kDa})$ in addition to two bands of higher molecular weight ( 46 and $49 \mathrm{kDa}$ ), one of which was determined to be due to phosphorylation of the protein. The protein is primarily detected in the cytoplasm of oocytes and is tightly regulated during meiotic and mitotic cell cycles. Progesterone stimulation of oocytes resulted in a rapid loss of Aven expression with the protein levels recovering before germinal vesicle breakdown (GVBD). This loss of Aven is required for the G2-M1 cell cycle transition. Aven morpholino knockdown experiments revealed that early depletion of the protein increases progesterone sensitivity and facilitates GVBD, but prolonged depletion of Aven results in caspase-3 activation and oocyte death by apoptosis. Phosphorylated Aven (46 kDa) was found to bind $\mathrm{Bcl}-\mathrm{x}_{\mathrm{L}}$ in oocytes, but this interaction was lost in apoptotic oocytes. Thus, Aven alters progesterone sensitivity in oocytes and is critical for oocyte survival.

Cell Death and Disease (2013) 4, e908; doi:10.1038/cddis.2013.435; published online 7 November 2013

Subject Category: Cancer

In order for oocytes to undergo fertilization, meiotic maturation is required, that is the progression from a G2 prophasearrested oocyte to an M phase-arrested oocyte. In Xenopus laevis oocytes, progesterone is used to stimulate resumption of the meiotic cell cycle, whereupon adenylyl cyclase activity is inhibited, cAMP levels are decreased and protein kinase $A$ (PKA) activity is suppressed. ${ }^{1-3}$ This decrease in PKA activity is necessary for synthesis of MOS protein and initiation of the MOS-MEK-MAPK signaling cascade and subsequent germinal vesicle breakdown (GVBD) ${ }^{4,5}$

Aven is an apoptotic regulator, inhibiting mitochondrial apoptosis by binding to and inhibiting the self-association of pro-apoptotic Apaf-1 and binding to and enhancing antiapoptotic $\mathrm{Bcl}-\mathrm{x}_{\mathrm{L}}$ activity. ${ }^{6-10}$ Overexpression of human Aven mRNA in Xenopus oocytes delays oocyte maturation, whereas overexpression of human and Drosophila Aven in Xenopus egg extract causes mitotic cell cycle arrest. ${ }^{11,12}$ Following DNA damage, it was shown that Aven activates ataxia telangiectasia-mutated (ATM) kinase to inhibit G2/M cell cycle progression. ${ }^{11}$

There is evidence for steroidal regulation of Aven. It has recently been shown that estrogen upregulates Aven expression at both the mRNA and protein level, in rat seminiferous tubules cultured ex vivo. ${ }^{13}$ Also, neonatal rat ovaries cultured in the presence of progesterone show decreased Aven gene expression in microarray analysis of the ovarian transcriptome. ${ }^{14}$
Aven has been linked to several diseases including cancer, Prader-Willi syndrome and amyotrophic lateral sclerosis. Aven gene expression is associated with poor prognosis in several cancers, including childhood acute lymphoblastic leukemia, acute myeloid leukemia ${ }^{15}$ and breast cancer. ${ }^{9}$ Microarray analysis has also identified Aven as being overexpressed in an ovarian carcinoma cell line resistant to the chemotherapeutic agent vincristine,${ }^{16}$ and underexpressed in colon cancer cell lines resistant to methotrexate. ${ }^{17}$

Here we present an analysis of endogenous Aven expression during oogenesis, oocyte maturation and early embryonic development in Xenopus laevis. We show that progesterone-stimulated maturation leads to an initial loss of Aven protein that is required for progression to GVBD with protein levels recovering thereafter. In addition, morpholino knockdown revealed that loss of Aven protein facilitated a rapid progression to GVBD but ultimately resulted in oocyte death by apoptosis.

\section{Results}

Aven expression and post-translational modification in oocytes and embryos. The predicted molecular weight of Xenopus Aven is $34.6 \mathrm{kDa}$ and an analysis of Aven expression in oocytes and embryos revealed a band close to the predicted molecular weight of the protein $(36 \mathrm{kDa})$ in

\footnotetext{
${ }^{1}$ UCD School of Bimolecular and Biomedical Science, UCD Conway Institute of Biomolecular and Biomedical Research, Belfield, Dublin 4, Ireland and ${ }^{2}$ UCD School of Agriculture, Food Science and Veterinary Medicine, UCD Conway Institute of Biomolecular and Biomedical Research, Belfield, Dublin 4, Ireland

*Corresponding author: C Hensey, UCD School of Biomolecular and Biomedical Science, UCD Conway Institute of Biomolecular \& Biomedical Research, University College Dublin, Belfield, Dublin 4, Ireland. Tel: +353 1716 6739; Fax: +353 1716 6456; E-mail: carmel.hensey@ucd.ie

Keywords: aven; meiosis; Xenopus; GVBD; apoptosis; oocyte

Abbreviations: ATM, ataxia telangiectasia-mutated; APAF-1, apoptotic protease activating factor-1; BCL- $\mathrm{x}_{\mathrm{L}}$, B-cell lymphoma-extra large; BSA, bovine serum albumin; CAMP, cyclic adenosine monophosphate; CDE, cell cycle-dependent element; CHR, cell cycle genes homology region; $\mathrm{CHO}$, chinese hamster ovary; GAPDH, Glyceraldehyde-3-phosphate dehydrogenase; GV, germinal vesicle; GVBD, germinal vesicle breakdown; HA, hemagglutinin; LR-NES, leucine-rich nuclear export sequence; MAPK, mitogen-activated protein kinases; MBT, mid blastula transition; MII, metaphase II; PKA, protein kinase A

Received 19.4.13; revised 17.7.13; accepted 11.9.13; Edited by M Piacentini
} 
addition to two bands of higher molecular weight (46 and $49 \mathrm{kDa}$ ) (Figure 1a). The 36 and $46 \mathrm{kDa}$ bands were detected throughout oogenesis and in pre- and post-mid blastula transition (MBT) embryos. The highest molecular weight 49-kDa band was only detected in previtellogenic stage II and III oocytes. Comparing nuclear and cytoplasmic fractions from stage VI oocytes, we demonstrated that Aven is entirely cytoplasmic (Figure 1b). This is in agreement with previous reports that Aven primarily localizes to the cytosol, whereas a small fraction is reported to be nuclear. ${ }^{6}$ Studies have shown that the intracellular localization of Aven is highly regulated and Aven contains a highly conserved leucine-rich nuclear export sequence (LR-NES). ${ }^{18}$

The intensity of the 36- and 46-kDa band varied, with levels decreasing as oogenesis proceeded. In pre-MBT embryos, the $46-\mathrm{kDa}$ band in particular fluctuated, this may reflect stage-dependent changes in the protein or cell cycledependent changes as embryos synchronously divide (Figure 1c). Post MBT in gastrulation stage embryos, the 46-kDa band was stabilized and predominated.

To verify that the antibody was in fact detecting Aven protein, we used an Aven morpholino oligonuceotide to inhibit Aven protein synthesis. Stage VI oocytes were microinjected $1 \mathrm{~h}$ before progesterone-stimulated in vitro maturation. Western blot analysis of GVBD samples showed the knockdown of both the $36-\mathrm{kDa}$ and the $46-\mathrm{kDa}$ bands, indicating that both bands represent the Aven protein (Figure 1d).

The Xenopus AVEN protein sequence has several predicted phosphorylation sites by multiple kinases (Supplementary Figure S1). Treatment of stage II and stage VI oocytes with potato acid phosphatase caused the depletion of the 46-kDa band after a 2-h incubation (Figure 1e). This indicates that $46-\mathrm{kDa}$ band represents a phosphorylated form of the AVEN protein.

Aven is degraded on progesterone stimulation and is tightly regulated during both meiotic and mitotic cell cycles. To determine whether Aven protein levels fluctuated during the meiotic cell cycle, we performed western blot analysis on oocytes undergoing progesterone-induced synchronous meiosis (Figure 2a). We consistently observed a complete loss of Aven expression within $30 \mathrm{~min}$ of progesterone stimulation. Aven levels always recovered within $1 \mathrm{~h}$ and before GVBD. Both the 36- and 46-kDa band were expressed for the remainder of oocyte maturation with a

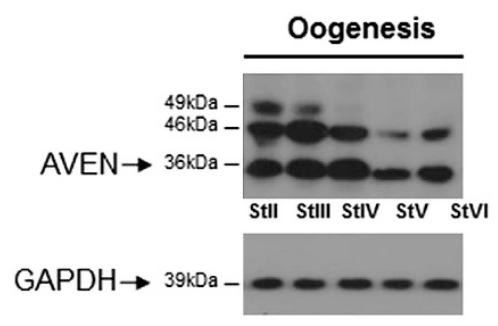

C

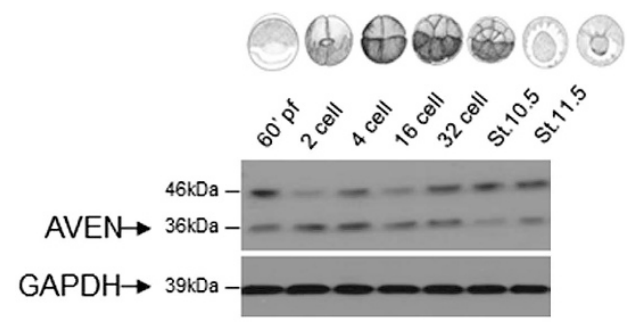

b

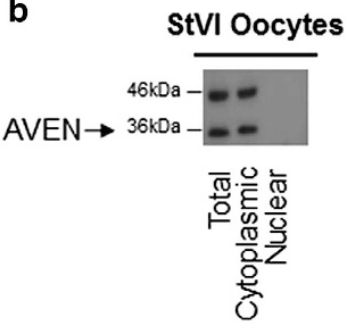

d

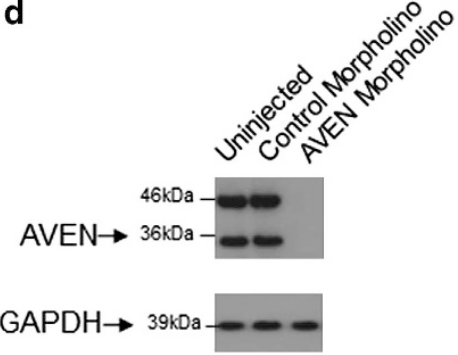

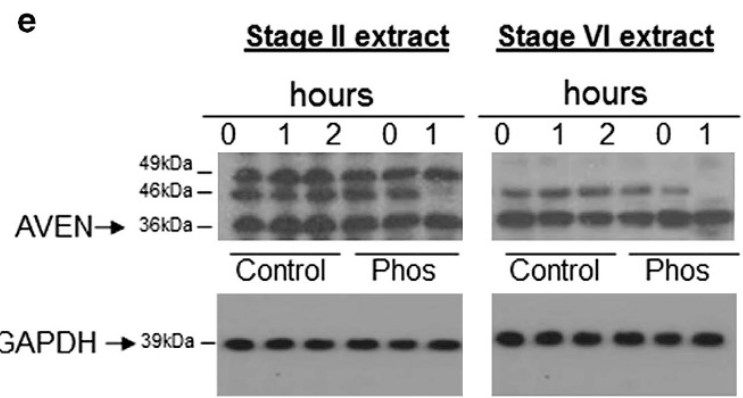

Figure 1 AVEN expression and subcellular localization in oocytes. (a) Representative western blot analysis of Aven expression in stage II-stage VI oocyte extracts. GAPDH was used as a loading control $(n=5)$. (b) Western blot analysis of stage VI whole oocyte extract, cytoplasmic extract and nuclear extract $(n=3)$. (c) Aven expression in embryos from single cell to stage $11.5(n=3)$. (d) Aven morpholino results in a loss of Aven expression at GVBD $(n=6)$. (e) Phosphatase treatment of Stage II and Stage $\mathrm{VI}$ oocytes caused depletion of the $46-\mathrm{kDa}$ Aven band, $(n=3)$ 

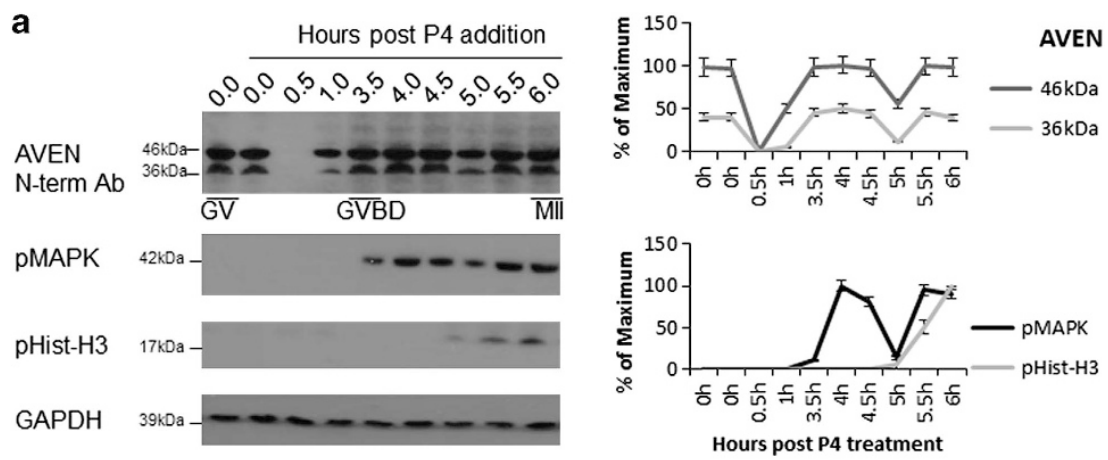

b
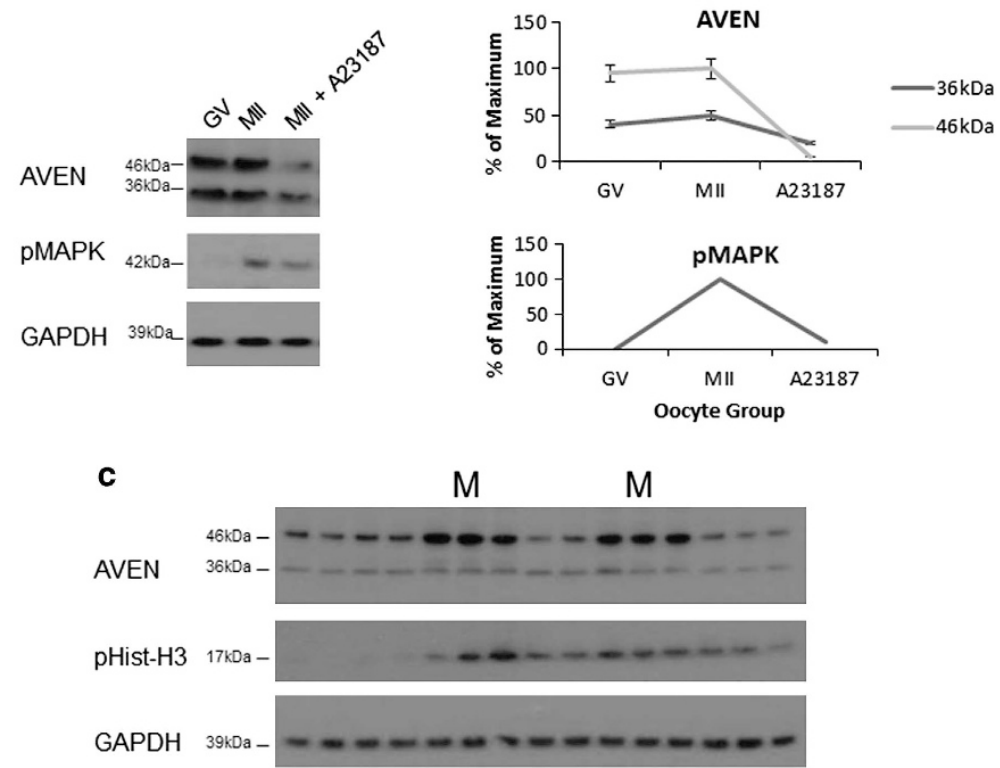

Figure 2 Dynamic changes in Aven expression during oocyte maturation. (a) Western blot analysis of Aven in oocyte extracts before and at the indicated time points (hours) after progesterone (P4) addition. In all, 64\% of oocytes reached GVBD by $3.5 \mathrm{~h}$ as determined by the appearance of a white spot (92\% at $4 \mathrm{~h}$ ) and GVBD and MII were confirmed by pMAPK and pHistone-H3 expression. Aven expression is rapidly lost on progesterone addition and recovers by GVBD, error bars correspond to S.D. based on four experiments. (b) Loss of Aven expression following calcium ionophore A23187 induced metaphase release, error bars correspond to S.D., $(n=4)$. (c) Fluctuations in Aven expression in cycling extracts with pHistone-H3 peaks marking the $\mathrm{M}$ phase of the cell cycle

the expression of both bands peaking at $\mathrm{Ml}$ and $\mathrm{MII}$, as determined by phospho MAPK levels. Calcium ionophore (A23187) induced metaphase II release, confirmed by pHistone-H3 depletion, resulted in a decrease in Aven expression (Figure 2b). Next, we examined Aven levels throughout the mitotic cell cycle using cycling extracts where oscillations were assessed by following pHistone- $\mathrm{H} 3$ expression. Aven protein levels, particularly the $46-\mathrm{kDa}$ band, appear to peak just before the $M$ phases of the cell cycle (Figure 2c).

\footnotetext{
Aven protein depletion increases progesterone sensitivity, facilitates GVBD but ultimately results in oocyte death. In progesterone-stimulated oocytes, antisense morpholino knockdown of Aven protein expression accelerated the rate of GVBD, scored by the appearance of a white spot on the top of the oocyte indicating the breakdown of the oocyte nucleus (Figure 3a). Oocytes lacking Aven showed 50\% GVBD more than an hour earlier than control morpholino-injected oocytes or uninjected
}

oocytes (Figures 3a and b). As phosphorylation of MAPK is essential for GVBD, we compared the timecourse of MAPK phosphorylation (pMAPK) in control and Aven morpholino-treated oocytes (Figure 3c). Following Aven knockdown, pMAPK was evident at $2 \mathrm{~h}$ compared with $3 \mathrm{~h}$ in control oocytes, indicating a more rapid progression to GVBD, correlated with an earlier activation of the signaling pathway. Aven knockdown had no effect on oocytes in the absence of progesterone (data not shown).

Oocytes injected with the Aven morpholino had increased sensitivity to progesterone, with 10-fold less progesterone needed to induce $50 \%$ GVBD by $5 \mathrm{~h}$ compared with control oocytes (Figure 3 e). Strikingly, at a very low concentration of progesterone $(5 \mu \mathrm{g} / \mathrm{ml}), 37 \%$ of oocytes injected with the morpholino showed GVBD compared with only $8 \%$ of the control oocytes.

Aven knockdown led to oocyte death following the onset of GVBD, with $50 \%$ of oocytes dying by $6 \mathrm{~h}$ post progesterone addition (Figure 3d). This oocyte death was characterized by the classic apoptotic phenotype previously described, ${ }^{19}$ 
a

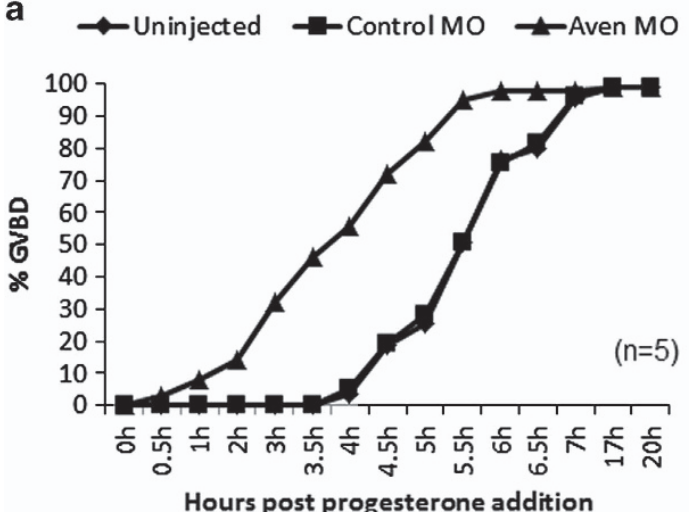

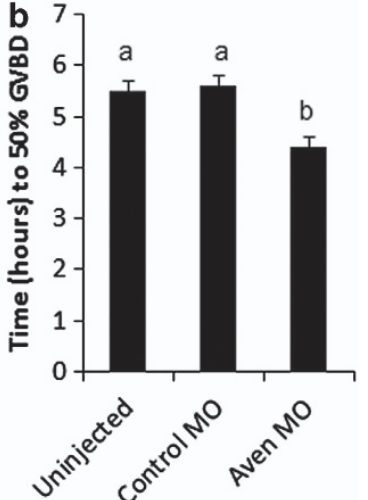

C
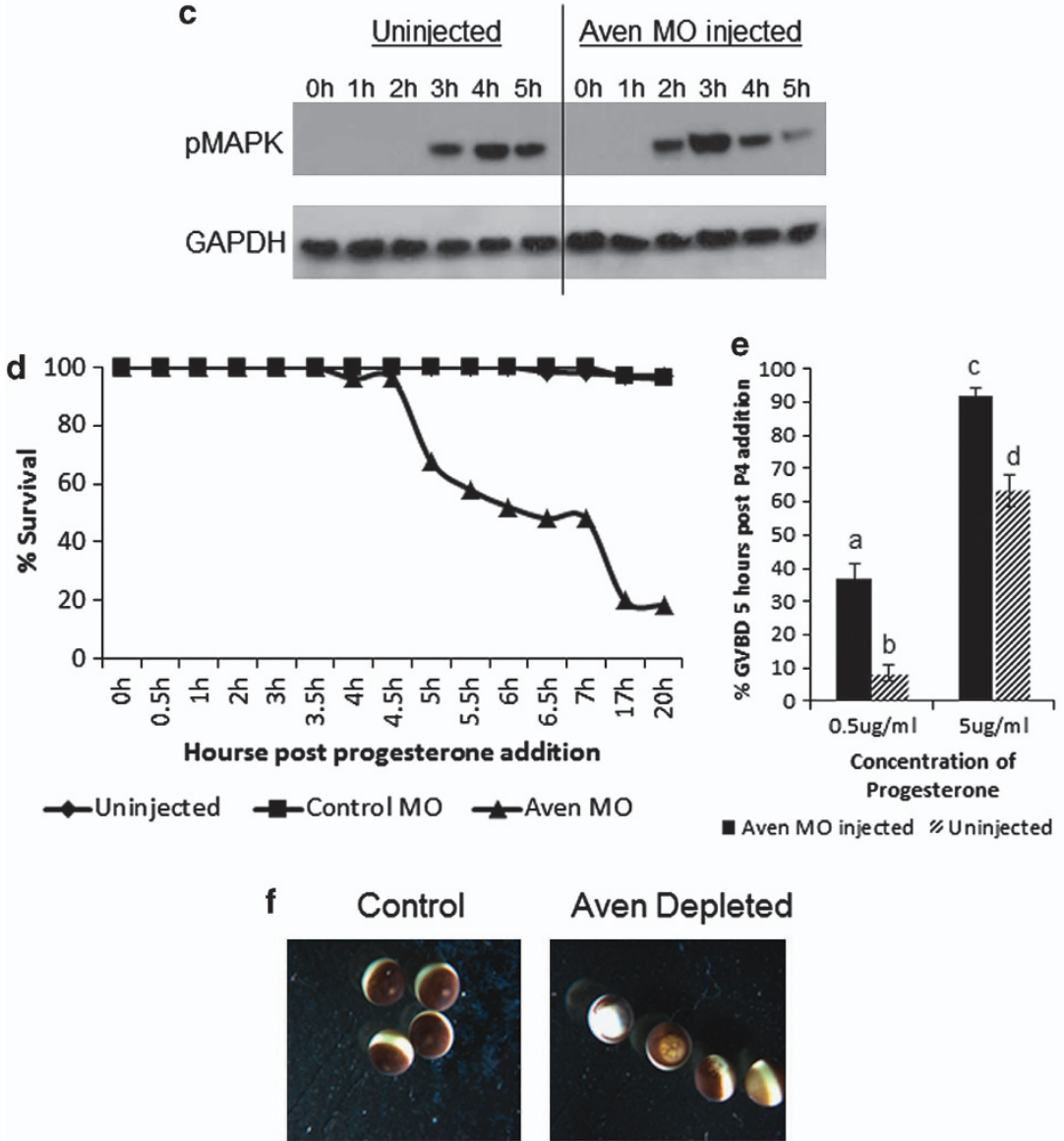

Aven Depleted

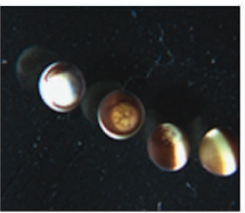

Figure 3 AVEN protein depletion in oocytes increases progesterone sensitivity, facilitates rapid GVBD but ultimately results in oocyte death. (a) \% GVBD in uninjected, control morpholino (MO)-injected and Aven morpholino (MO)-injected progesterone-treated oocytes (80-120 oocytes per experiment). Shown is a representative experiment from $n=5$. (b) Columns represent the average time taken for $50 \%$ GVBD to occur; error bars correspond to S.D., ( $n=5$ ). (c) Western blot analysis of pMAPK expression in uninjected and AVEN MO-injected oocytes at the indicated times after progesterone addition. pMAPK is evident $1 \mathrm{~h}$ earlier in Aven MO-treated oocytes. (d) Oocyte survival was scored at the indicated times in uninjected, control MO and AVEN MO-injected oocytes. Aven MO-treated embryos gradually die as determined by gross morpholoigcal features. (e) Columns represent \% GVBD in uninjected and Aven MO-injected oocytes $5 \mathrm{~h}$ after addition of the indicated progesterone concentrations. Loss of Aven increases the sensitivity of oocytes to progesterone. Error bars correspond to S.D., $(n=5)$. (f) The apoptotic morphology of Aven-depleted oocytes as compared with control oocytes represented in d. Columns with different letters indicate significant differences $(P<0.05)$

where the oocytes displaced pigment granules to form a large white spot at the animal pole of the oocyte (Figure 3f).

Aven promotes oocyte survival by interacting with $\mathrm{BCl}-\mathrm{x}_{\mathrm{L}}$ and preventing apoptosis. To assess whether the oocyte death induced by Aven protein depletion is due to apoptosis, morpholino-injected oocytes were examined by western blot for the presence of active caspase-3 (Figure 4a). Aven morpholino-injected oocytes showed activated caspase- 3 by $5 \mathrm{~h}$ after progesterone addition, 
whereas no caspase-3 activation was detected in uninjected or control morpholino-injected oocytes. This supports activation of an apoptotic pathway in Avendepleted oocytes, as indicated by the visual morphology (Figure 4b).

The interaction between Aven and $\mathrm{Bcl}-\mathrm{x}_{\mathrm{L}}$ is important in promoting cell survival, ${ }^{8}$ therefore, we compared Aven-Bcl- $x_{L}$ interaction in normal and DNA damageinduced apoptotic oocytes. Progesterone-stimulated and $\gamma$-irradiated oocytes underwent apoptosis at a time equivalent to MII as indicated by caspase-3 activation(Figure 4c, input $\mathrm{MII}^{*}$ ). Despite the decreased Aven expression in apoptotic oocytes, it is possible to concentrate the remaining $46-\mathrm{kDa}$ form of the protein by immunoprecipitation and $\mathrm{Bcl}-\mathrm{x}_{\mathrm{L}}$ co-immunoprecipitates with this (Figure 4c, Aven IP). Similar levels of $\mathrm{Bcl}-\mathrm{x}_{\mathrm{L}}$ from both normal and apoptotic oocyte samples were immunoprecipitated, however significantly less Aven protein was associated with $\mathrm{Bcl}-\mathrm{x}_{\mathrm{L}}$ in the apoptotic oocytes (Figure 4c, Bcl- $x_{L} I P$ ). This loss of Aven interaction with $\mathrm{Bcl}-\mathrm{x}_{\mathrm{L}}$ in apoptotic oocytes points to the significance of this interaction at GVBD and MII for oocyte survival.

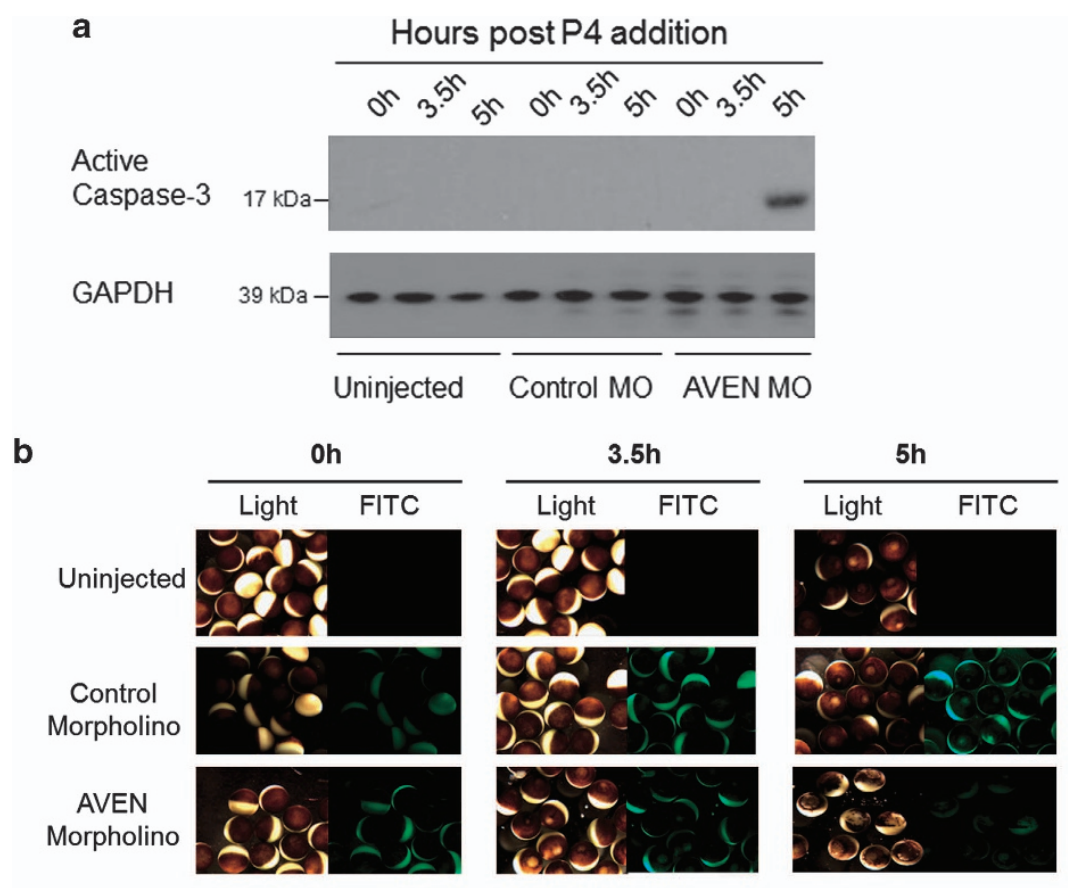

c

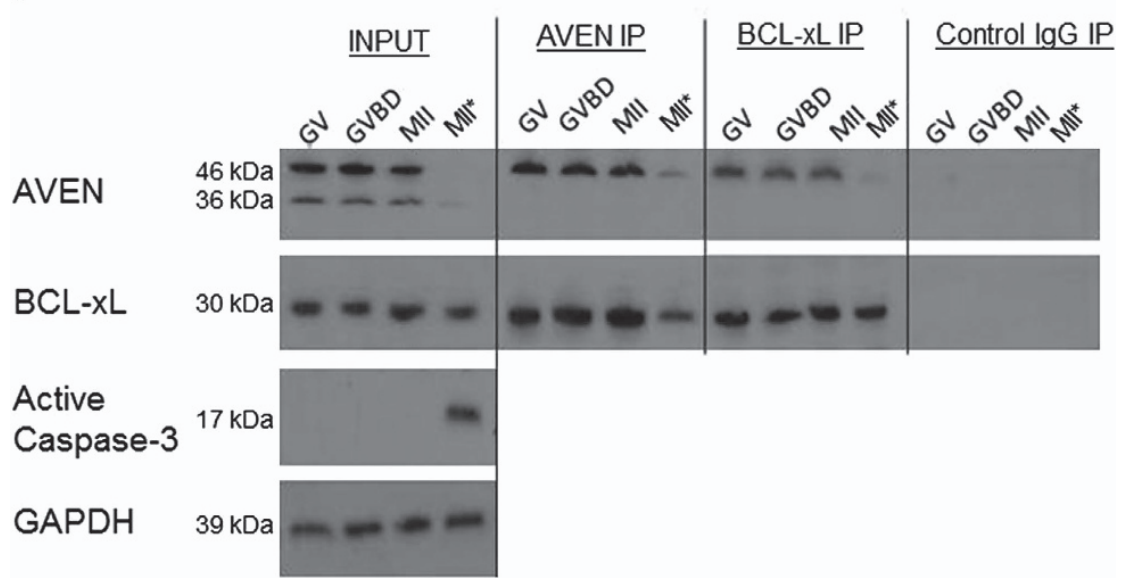

Figure 4 Cell death activation in Aven-depleted oocytes. (a) Western blot analysis for active caspase-3 expression in uninjected, control MO-injected and Aven MO-injected, progesterone-treated oocytes (100-120 oocytes per experiment). Active caspase- 3 was only detected in Aven-depleted oocytes $5 \mathrm{~h}$ after progesterone treatment. Shown is a representative experiment from $n=7$. (b) Representitive images (light and fluorescence) of Aven MO-injected oocytes undergoing precocious GVBD and subsequent apoptosis at the indicated times after progesterone addition. (c) A comparison of Aven-Bcl- $\mathrm{X}_{\mathrm{L}}$ interaction in control and apoptotic oocytes. Aven and Bcl- $\mathrm{X}_{\mathrm{L}}$ immunoprecipates were analyzed by western blot at GV, GVBD, MII and MII*: an MII equivalent time point in irradiated oocytes $(n=3)$. Normal rabbit lgG antibody was used in control immunoprecipitations. Bcl- $x_{L}$ is bound to Aven in immoprecipiated MII-arrested egg extract, whereas in DNA damage-induced apoptotic extracts, little Aven is assoictaed with $\mathrm{BCl}-\mathrm{x}_{\mathrm{L}}$ 


\section{Discussion}

To date, the only reported endogenous expression of Aven indicates a protein with a higher molecular weight than predicted; analysis of human Aven expression in HeLa cells identified a protein with an apparent molecular weight of $55 \mathrm{kDa}$, even though the predicted molecular weight of the human protein is $38.6 \mathrm{kDa} .{ }^{6} \mathrm{~A}$ recombinant HA-tagged Drosophila Aven yielded a protein of $\sim 36 \mathrm{kDa}$, excluding the HA tag. ${ }^{12}$ The detection of a $36-k D a$ band in Xenopus oocytes and embryos is the first report of endogenous Aven close to its predicted molecular weight. The bands of higher apparent molecular weight detected in Xenopus may represent a similar form to that reported in humans and in all likelihood these represent post-translationally modified forms of Aven. Recombinant Aven expression in a Chinese hamster ovary $(\mathrm{CHO})$ cell line resulted in four closely separated bands being detected by western blot, providing further evidence that Aven is post-transitionally modified. ${ }^{20}$

Human Aven is a known substrate for the ATM kinase at two SQ sites and evidence of additional basal phosphorylation at Ser and Thr sites also exists. ${ }^{11}$ Treatment of stage II and stage VI oocytes with potato acid phosphatase caused the depletion of the $46-\mathrm{kDa}$ band after a $2-\mathrm{h}$ incubation (Figure 1e), indicating that this band represents a phosphorylated form of the Aven protein. The 10-kDa molecular weight shift suggests hyperphosphorylation of the protein at multiple sites. Analysis of the protein sequence reveals several different kinase phosphomotifs, including potential CK2, PKA and an ATM site, ${ }^{11,21}$ (Supplementary Figure S1a).

The phosphomotifs identified are putative substrates for kinases that are known regulators of the cell cycle and/or oocyte maturation. PKA, in particular, is a negative regulator of the G2/M1 transition, and is rapidly depleted in response to progesterone stimulation. It has long been postulated that this decrease in PKA activity results in decreased phosphorylation of a presumptive maturation-inhibiting phosphoprotein. ${ }^{22,23}$ Aven would be a candidate for such a protein if phosphorylation by PKA stabilized the protein preventing GVBD.

The $49-\mathrm{kDa}$ band in stage II extracts was resistant to phosphatase treatment, suggesting that either different posttranslational modifications are altering the proteins apparent molecular weight or there are additional isoforms of the protein present. Studies on human and mouse Aven have indeed considered the possibility of different isoforms being present. Northern blot analysis detected a single 1.7-kb Aven transcript in all human adult tissues tested, with highest expression in heart, skeletal muscle, kidney, liver, pancreas and testis and a number of cell lines. ${ }^{6}$ Analysis of the mouse Aven gene revealed two isoforms, a short (mAven-S) and a longer ( $m A$ ven- $L$ ) transcript (corresponding to the Aven gene found in other species) encoding proteins of 216 and 342 amino acids, respectively. ${ }^{24}$ Thus, there is no evidence from other species studied that the $49-55 \mathrm{kDa}$ protein identified represents a different isoform of the protein. The Aven sequence shows putative $\mathrm{O}$ and $\mathrm{N}$ glycosylation sites, and it will be interesting to investigate whether such post-translational modifications are responsible for the highest molecular weight forms of the protein.
The expression of Aven as two bands of 36 and $46 \mathrm{kDa}$ is evident at different stages during meiotic maturation. Downregulation of the protein is evident at two points; immediately following progesterone stimulation and a less significant reduction in protein levels between $\mathrm{MI}$ and $\mathrm{MII}$. It is of note that there is no evidence of a classical destruction box in the Aven sequence. However, there are several different mechanisms by which proteins might be rapidly degraded, including ubiquitin-mediated proteasomal degradation and proteolytic cleavage. Our preliminary analysis using a proteasome inhibitor suggests proteasomal degradation does not have a role in Aven degradation. The CK2-regulated caspase 8/9 site identified in human Aven is not conserved in Xenopus, ruling out cleavage by these caspases. ${ }^{21}$ Aven cleavage by Cathepsin $D$ is important for the anti-apototic potential of the protein and the Cathepsin D cleavage sites are conserved across species, including Xenopus. ${ }^{25}$ Using an antibody directed against the C-terminal of the Aven protein, we have been able to detect a band with an apparent molecular weight of $28 \mathrm{kDa}$, this could represent the predicted Cathepsin D Aven fragment (Supplementary Figure S1b). This fragment is not degraded on progesterone treatment and any generation of a fragment by Cathepsin $D$ activity is more likely related to oocyte survival rather than the required loss of the higher molecular weight forms for G2-MI progression following progesterone treatment.

During meiosis, Aven is consistently associated with BCL$\mathrm{X}_{\mathrm{L}}$ and specifically the $46-\mathrm{kDa}$ phosphorylated form of the protein. This suggests that the $B C L-x_{L}$ interaction is dependent on particular post-translational modification(s) of the protein and only a subpopulation of the AVEN interacts with BCL-X $x_{L}$.

In silico analysis of the Aven promoter region, using Matbase from Genomatix (http://www.genomatix.de), enabled the identification of a progesterone receptor-binding site and potential CDE/CHR cell-dependent element, which is known to regulate cell cycle-dependent transcription ${ }^{26}$ (Supplementary Figure S1c). The role of progesterone in regulating Aven warrants further study and while cell cycle regulation may not be relevant during oocyte maturation, it may have a role in the mitotic cell cycle regulation of Aven observed within the embryo.

Modulation of Aven expression initially impacts on G2-Ml progression and ultimately has consequences for oocyte survival. The mechanism by which the progesterone induced cytoplasmic signaling pathway that leads to oocyte maturation impacts on Aven expression remains to be elucidated. It is known that Aven can prevent M-phase entry following DNA damage through its ability to activate ATM and overexpression of the protein inhibits G2/M in oocytes. ${ }^{11}$ Our analysis of endogenous Aven expression shows how its loss is required for this cell cycle transition to occur, suggesting that Aven has the capacity to interfere with cell cycle progression independent of a DNA damage signal. Recovery of Aven protein levels is required for oocyte viability, highlighting Aven's importance as an apoptotic inhibitor in the maturing oocyte. Aven clearly has a dual role in regulating both cell cycle progression and oocyte survival and whether these activities are integrated or are underpinned by completely separate biochemical activities requires further analysis. 


\section{Materials and Methods}

Xenopus oocyte isolation and embryo culture. All animal experiments were conducted under license from the Department of Health and Children (B100/3003) and according to protocols approved by the University College Dublin Animal Research Ethics Committee (AREC-P-10-68).

Stage II-VI oocytes ${ }^{27}$ for oogenesis experiments were obtained via collagenase defolliculation ( $2 \mathrm{mg} / \mathrm{ml}$, Sigma, St. Louis, MO, USA). Stage VI oocytes were obtained by manual defolliculation, matured with progesterone (Sigma; P7556) at the indicated concentrations, observed for GVBD and nuclear extracts were made as described ${ }^{28,29}$ For phosphatase treatment, St II and St VI oocyte extracts were incubated with potato acid phosphatase at $0.5 \mu \mathrm{g} / \mu \mathrm{l}$ extract. ${ }^{30}$ Calcium ionophore A23187 (Sigma, C7522) was added to OR2 culture media at a concentration of $2 \mu \mathrm{g} / \mathrm{ml}$ to activate metaphase II-arrested oocytes. ${ }^{31}$ In vitro fertilization was carried out as described. ${ }^{32}$ Embryos were staged, ${ }^{33}$ collected and snap-frozen in liquid nitrogen at the indicated stages. Cycling extracts were prepared from unfertilized eggs activated using calcium ionophore A23187 (Sigma; C7522), as described in Murray. ${ }^{34}$ Samples were collected at 10-min intervals and snap-frozen in liquid nitrogen.

$\gamma$-Irradiation. Irradiation of oocytes and embryos was performed using a Gamma Cell Cs ${ }^{137}$ source. Irradiated samples (33 Gy) were subsequently treated with progesterone to induce maturation.

Aven knockdown. Fluoresceine-tagged Aven morpholino (5'-GGCCC CGCTCCATGTGTTCGGTATC-3') and control morpholino (5'-CCTCTTACCT CAGTTACAATTTATA- $3^{\prime}$ ) were obtained from Gene Tools, LLC (Philomath, OR, USA) (http://www.gene-tools.com). The morpholinos were microinjected into stage VI oocytes, at a final concentration of $5 \mu \mathrm{M}, 1 \mathrm{~h}$ before progesterone treatment. To confirm microinjection, live oocytes were observed for fluorescence using a Carl Zeiss Ltd. (Cambridge, UK) stereo Lumar V12 with FITC filter.

Immunoblotting, imuunoprecipitation and antibodies. Protein samples were separated according to their apparent molecular mass on NuPAGE 4-12\% Bis-Tris Gels (Invitrogen, Carlsbad, CA, USA), and transferred to a PVDF membrane. After blocking in $5 \%$ bovine serum albumin (BSA) in PBS- $0.1 \%$ Tween-20, membranes were incubated with the primary antibody, followed by incubation with horseradish peroxidase-conjugated secondary antibodies. Proteins were visualized using Enhanced Chemiluminescence Western Blotting substrate (Thermo Scientific, Rockford, IL, USA; \#32106). Densitometry was performed using the ImageJ program (http://rsbweb.nih.gov/ij/).

Endogenous Aven was immunoprecipitated by incubating $40 \mu \mathrm{l}$ of oocyte extract with $20 \mu \mathrm{l}$ of Aven antibody or control normal rabbit lgG antibody overnight at $4{ }^{\circ} \mathrm{C}$, then incubating with protein $A / G$ agrose beads (Thermo Scientific, 20421) for $2 \mathrm{~h}$ at room temperature. Washes were performed using IP buffer ( $25 \mathrm{mM}$ Tris, $150 \mathrm{mM}$ $\mathrm{NaCl} ; \mathrm{pH} 7.2)$

Anti-AVEN antibodies (Cell Signaling Technology Inc., Danvers, MA, USA \#2300; Abcam, Cambridge, UK, ab77014), anti-phospho-histone H3 (Ser10) antibody (Merck Millipore, Bitterica, MA, USA, cat. \#06-570), anti-GAPDH antibody (Cell Signaling, \#3900), anti-pMapk (Cell Signaling, \#9101), antiCaspase-3 (BD BioSciences, Oxford, UK, \#559565), anti-Bcl-x L (Santa Cruz Biotechnology, Santa Cruz, CA, USA, sc-7195), normal Rabbit IgG (Cell Signaling, \#2729).

Two antibodies raised against an $\mathrm{N}$ - and C-terminal epitope in human Aven were compared for their ability to detect Xenopus Aven (Supplementary Figure S1b). The N-terminal antibody detected bands of 36 and $46 \mathrm{kDa}$, both of which were knocked down following injection of Aven morpholino to oocytes (Figure 1d). This antibody was used throughout the study. The C-terminal antibody also detected the 46-kDa band and in addition a $28-\mathrm{kDa}$ band, which may be the previously reported cathepsin D-cleaved C-terminal proteolytic fragment. ${ }^{25}$

Statistical analysis. Statistical analyses were carried out using the GraphPad Prism version 6.0 software package for Windows. Differences between groups were considered significant when $P$-values were less than 0.05 .

\section{Conflict of Interest}

The authors declare no conflict of interest.
Acknowledgements. This work is funded by Science Foundation Ireland, grant number 07/SRC/B1156.

1. Maller JL, Krebs EG. Progesterone-stimulated meiotic cell division in Xenopus oocytes. Induction by regulatory subunit and inhibition by catalytic subunit of adenosine 3':5'-monophosphate-dependent protein kinase. J Biol Chem 1977; 252: 1712-1718

2. Finidori-Lepicard J, Schorderet-Slatkine S, Hanoune J, Baulieu EE. Progesterone inhibits membrane-bound adenylate cyclase in Xenopus laevis oocytes. Nature 1981; 292 : 255-257.

3. Sadler SE, Maller JL. Progesterone inhibits adenylate cyclase in Xenopus oocytes. Action on the guanine nucleotide regulatory protein. I Biol Chem 1981; 256: 6368-6373.

4. Daar I, Yew N, Vande Woude GF. Inhibition of mos-induced oocyte maturation by protein kinase A. J Cell Biol 1993; 120: 1197-1202.

5. Matten W, Daar I, Vande Woude GF. Protein kinase A acts at multiple points to inhibit Xenopus oocyte maturation. Mol Cell Biol 1994; 14: 4419-4426.

6. Chau BN, Cheng EH, Kerr DA, Hardwick JM. Aven a novel inhibitor of caspase activation, binds Bcl-xL and Apaf-1. Mol Cell 2000; 6: 31-40.

7. Dorai $\mathrm{H}$, Ellis D, Keung YS, Campbell M, Zhuang M, Lin C et al. Combining high-throughput screening of caspase activity with anti-apoptosis genes for development of robust $\mathrm{CHO}$ production cell lines. Biotechnol Progress 2010; 26: 1367-1381.

8. Figueroa B Jr, Chen S, Oyler GA, Hardwick JM, Betenbaugh MJ. Aven and Bcl-xL enhance protection against apoptosis for mammalian cells exposed to various culture conditions. Biotechnol Bioeng 2004; 85: 589-600.

9. Kutuk O, Temel SG, Tolunay S, Basaga H. Aven blocks DNA damage-induced apoptosis by stabilising Bcl-xL. Eur J Cancer 2010; 46: 2494-2505.

10. Zou H, Li Y, Liu X, Wang X. An APAF-1.cytochrome c multimeric complex is a functional apoptosome that activates procaspase-9. J Biol Chemistry 1999; 274: 11549-11556.

11. Guo JY, Yamada A, Kajino T, Wu JQ, Tang W, Freel CD et al. Aven-dependent activation of ATM following DNA damage. Curr Biol 2008; 18: 933-942.

12. Zou S, Chang J, LaFever L, Tang W, Johnson EL, Hu J et al. Identification of dAven, a Drosophila melanogaster ortholog of the cell cycle regulator Aven. Cell Cycle 2011; 10: 989-998.

13. Laurentino S, Goncalves J, Cavaco JE, Oliveira PF, Alves MG, de Sousa M et al. Apoptosis-inhibitor Aven is downregulated in defective spermatogenesis and a novel estrogen target gene in mammalian testis. Fertil Steril 2011; 96: 745-750.

14. Nilsson EE, Stanfield J, Skinner MK. Interactions between progesterone and tumor necrosis factor-alpha in the regulation of primordial follicle assembly. Reproduction 2006; 132: 877-886

15. Paydas S, Tanriverdi K, Yavuz S, Disel U, Sahin B, Burgut R. Survivin and aven: two distinct antiapoptotic signals in acute leukemias. Annals Oncol 2003; 14: 1045-1050.

16. Buys TP, Chari R, Lee EH, Zhang M, MacAulay C, Lam S et al. Genetic changes in the evolution of multidrug resistance for cultured human ovarian cancer cells. Genes Chromosomes Cancer 2007; 46: 1069-1079.

17. Selga E, Oleaga C, Ramirez S, de Almagro MC, Noe V, Ciudad CJ. Networking of differentially expressed genes in human cancer cells resistant to methotrexate. Genome Med 2009; 1: 83.

18. Esmaili AM, Johnson EL, Thaivalappil SS, Kuhn HM, Kornbluth S, Irusta PM. Regulation of the ATM-activator protein Aven by CRM1-dependent nuclear export. Cell Cycle 2010; 9 : 3913-3920.

19. Nutt LK, Margolis SS, Jensen M, Herman CE, Dunphy WG, Rathmell JC et al. Metabolic regulation of oocyte cell death through the CaMKII-mediated phosphorylation of caspase-2. Cell 2005; 123: 89-103.

20. Sauerwald TM, Figueroa B Jr, Hardwick JM, Oyler GA, Betenbaugh MJ. Combining caspase and mitochondrial dysfunction inhibitors of apoptosis to limit cell death in mammalian cell cultures. Biotechnol Bioeng 2006; 94: 362-372.

21. Turowec JP, Duncan JS, Gloor GB, Litchfield DW. Regulation of caspase pathways by protein kinase CK2: identification of proteins with overlapping CK2 and caspase consensus motifs. Mol Cell Biochem 2011; 356: 159-167.

22. Smith LD. The induction of oocyte maturation: transmembrane signaling events and regulation of the cell cycle. Development 1989; 107: 685-699.

23. Shibuya EK. G2 cell cycle arrest-a direct link between PKA and Cdc25C. Cell Cycle 2003; 2: $39-41$

24. Ina S, Tsunekawa N, Nakamura A, Noce T. Expression of the mouse Aven gene during spermatogenesis, analyzed by subtraction screening using Mvh-knockout mice. Gene Expr Patterns 2003; 3: 635-638.

25. Melzer IM, Fernandez SB, Bosser S, Lohrig K, Lewandrowski U, Wolters D et al. The Apaf-1-binding protein Aven is cleaved by Cathepsin D to unleash its anti-apoptotic potential. Cell Death Differ 2012; 19: 1435-1445

26. Lange-zu Dohna C, Brandeis M, Berr F, Mossner J, Engeland K. A CDE/CHR tandem element regulates cell cycle-dependent repression of cyclin B2 transcription. FEBS Lett 2000; 484: 77-81.

27. Dumont JN. Oogenesis in Xenopus laevis (Daudin). I. Stages of oocyte development in laboratory maintained animals. J Morphol 1972; 136: 153-179. 
28. Evans JP, Kay BK. Biochemical fractionation of oocytes. Methods Cell Biol 1991; 36: 133-148.

29. Smith LD, Xu WL, Varnold RL. Oogenesis and oocyte isolation. Methods Cell Biol 1991; 36: 45-60.

30. Walker J, Dale M, Standart N. Unmasking mRNA in clam oocytes: role of phosphorylation of a 3' UTR masking element-binding protein at fertilization. Dev Biol 1996; 173: 292-305.

31. Liu J, Grimison B, Lewellyn AL, Maller JL. The anaphase-promoting complex/cyclosome inhibitor Emi2 is essential for meiotic but not mitotic cell cycles. J Biol Chem 2006; 281: 34736-34741.

32. Heasman J, Holwill S, Wylie CC. Fertilization of cultured Xenopus oocytes and use in studies of maternally inherited molecules. Methods Cell Biology 1991; 36: 213-230.
33. Nieuwkoop P, Faber J. Normal table of Xenopus laevis (Daudin). Garland: Hamden, CT, 1994.

34. Murray AW. Cell cycle extracts. Methods Cell Biol 1991; 36: 581-605.

cc)(1) Cell Death and Disease is an open-access journal published by Nature Publishing Group. This work is licensed under a Creative Commons Attribution-NonCommercialShareAlike 3.0 Unported License. To view a copy of this license, visit http://creativecommons.org/licenses/by-nc-sa/3.0/

Supplementary Information accompanies this paper on Cell Death and Disease website (http://www.nature.com/cddis) 